\title{
Determinantes del ahorro de los hogares en México: un análisis de regresión cuantílica
}

\author{
Determinants of Household Savings in Mexico: \\ A Quantile Regression Analysis
}

\author{
Isalia Nava Bolaños ${ }^{*}$ Flor Brown Grossman ${ }^{* * *}$
}

\begin{abstract}
RESUMEN
Este artículo analiza los factores económicos y sociodemográficos explicativos del ahorro de los hogares en México. A diferencia de otras investigaciones, toma en cuenta diferentes puntos de la distribución de la tasa de ahorro. La metodología involucra la estimación de regresiones cuantílica y cuantílica con variables instrumentales, a partir de la Encuesta Nacional de Ingresos y Gastos de los Hogares 2014. Los resultados muestran las diferencias de factores explicativos a lo largo de la distribución de la tasa de ahorro. La propensión marginal al ahorro fue de 5.7 por ciento en QR20 y de 16.1 por ciento en QR90. El sexo de la jefatura y el acceso a los mercados financieros no tienen un efecto significativo en QR20. El número de personas mayores aumenta la tasa de ahorro en QR20 y QR50, pero no es significativa en los otros cuantiles. El efecto de la localidad urbana disminuye entre cuantiles, hasta volverse negativo en QR90.
\end{abstract}

Palabras clave: Comportamiento del ahorro, hogares, ingreso permanente, desigualdad, regresión cuantílica con variables instrumentales.

Clasificación JEL: D14, D15, E21, C21.

\begin{abstract}
This article analyses the economic and sociodemographic factors that explain household savings in Mexico. Unlike other studies, it takes into account different points of the distribution of saving rates. The methodology includes a quantile regression and instrumental variable quantile regression, based on the National Household Income and Expenditure Survey 2014. The results show the differences of determinants along the distribution of saving rates. The marginal propensity to save was 5.7 per cent in QR20 and 16.1 per cent in QR90. The sex of the household head and access to financial markets do not have a significant effect on QR20. The number of older people increases the saving rates in QR20 and QR50, but it is not significant in the other quantiles. The effect of urban areas decreases between quantiles, until it becomes negative in QR90.
\end{abstract}

Keywords: Saving behavior, housing, permanent income, inequality, instrumental variable quantile regression.

JEL Classification: D14, D15, E21, C21.

\footnotetext{
* Fecha de recepción: 02/03/2018. Fecha de aprobación: 19/06/2018.

** Universidad Nacional Autónoma de México. Correo electrónico: isalia@unam.mx. ORCID: 0000-0001-8317-4601.

*** Universidad Nacional Autónoma de México. Correo electrónico: brown@unam.mx. ORCID: 0000-0001-9943-6741.
} 


\section{INTRODUCCIÓN}

El ahorro, en su acepción más general en la bibliografía económica, se refiere a aquella parte del ingreso que no se destina al consumo, en la que interviene la dimensión temporal que obliga al agente económico a comparar el consumo al que se renuncia en el presente con aquel que se puede obtener en el futuro.

Según sectores institucionales, en el año 2016 el ahorro de los hogares en México representó 63.7 por ciento del ahorro bruto total de la economía, seguido por las sociedades no financieras con 24.2 por ciento. Al descomponer el ahorro interno, los hogares también aparecen como el sector institucional más importante; el monto de ahorro generado representó 15.5 por ciento en términos del Producto Interno Bruto (PIB), mientras que el de las sociedades no financieras fue 3.7 por ciento (INEGI, 2017).

Dada la relevancia que tienen los hogares, el énfasis de esta investigación se centra en su ahorro. La revisión bibliográfica permite corroborar que existen pocos estudios al respecto. Se considera que el conocimiento más profundo sobre los factores que determinan el comportamiento del ahorro en los hogares puede contribuir a la identificación de medidas y acciones encaminadas a movilizar recursos económicos para incrementarlo.

El objetivo de este documento es analizar los factores económicos y sociodemográficos explicativos del ahorro de los hogares en México. A partir de los microdatos de la Encuesta Nacional de Ingresos y Gastos de los Hogares 2014 (ENIGH), se estima un modelo de regresión cuantílica. En este trabajo, siguiendo de cerca a Chen, Kuan y Lin (2007) y a diferencia de otras investigaciones recientes para el caso de México (Nava, Brown y Domínguez, 2014; Valles y Aguilar, 2015), en las cuales no se reflexiona sobre la heterogeneidad de la propensión del ahorro de los hogares, dedicamos especial atención a los efectos del ingreso permanente y a su posible endogeneidad, a partir de la estimación de un modelo de regresión cuantílica con variables instrumentales.

Se proponen las siguientes preguntas de investigación: ¿Cuáles son las variables explicativas del ahorro de los hogares? ¿Cuál es el efecto de variables económicas, como el ingreso permanente, el acceso a mercados financieros, la presencia de redes de transferencias y el número de integrantes en el hogar sobre el ahorro? ¿Cuál es el efecto de variables sociodemográficas, como el sexo y la edad del jefe del hogar, la clase de hogar, el número de personas menores y mayores, y la localidad de residencia sobre el ahorro de los hogares? ¿Cuáles son las variables que tienen un efecto diferenciado en los diferentes puntos de la distribución de la tasa de ahorro?

El documento se divide en cuatro secciones. La primera describe de manera breve los principales antecedentes teóricos y de investigación sobre los 
determinantes del ahorro en México. La segunda presenta la fuente de información y la operacionalización de la variable "ahorro". La siguiente sección está dedicada a los resultados sobre el comportamiento de la tasa de ahorro según distintas características de la población, las estimaciones de la regresión cuantílica (QR20, QR50, QR60 y QR90), y las comparaciones de los resultados de la regresión cuantílica con los de la estimación con variables instrumentales. La última sección da cuenta de las conclusiones.

\section{ASPECTOS FUNDAMENTALES ACERCA DE LOS DETERMINANTES DEL AHORRO}

En la literatura en torno a los determinantes del ahorro prevalecen dos planteamientos teóricos: la hipótesis de ciclo de vida (HCV) propuesta por Modigliani y Brumberg (1954) y la hipótesis del ingreso permanente (HIP), desarrollada por Friedman (1957). Ambas suponen que los agentes económicos planean su consumo a largo plazo y que el ahorro depende de los ingresos esperados en el futuro. $\mathrm{El}$ ahorro disminuye si el nivel de ingreso es menor al previsto y aumenta en caso contrario. La diferencia más clara entre ambas hipótesis se refiere a cómo consideran las fluctuaciones del ingreso y su influencia sobre el consumo y el ahorro.

La HCV, al estar basada en un modelo de optimización intertemporal, permite localizar los patrones de ahorro a lo largo de la vida. En general, se identifican tres grandes etapas: juventud, edad laboral y retiro. En los años de juventud, cuando los ingresos son bajos, las personas piden préstamos para satisfacer sus necesidades de consumo. Más tarde, en la edad laboral, cuando se logran mejores niveles de ingreso, pagan las deudas adquiridas y proceden a la acumulación de recursos, anticipándose al futuro. En el retiro, los activos acumulados se reducen paulatinamente. De esta forma el ahorro describe un patrón con forma de U invertida a lo largo de la vida (Modigliani, 1986).

La HIP parte del supuesto de que tanto el ingreso como el consumo tienen dos componentes, uno permanente y otro transitorio (Friedman, 1957). Generalmente, la parte del ingreso que es permanente se destina al consumo, obviando las variaciones del ingreso transitorio. Por lo tanto, el ahorro será mayor cuando el ingreso transitorio sea más elevado (Liberda et al., 2004).

Un conjunto de estudios confirman ambas hipótesis al encontrar una relación positiva entre el ahorro de los hogares y el ingreso (Dynan, Skinner y Zeldes, 2004; Poterba, 1994). Sin embargo, estas hipótesis pueden ser limitadas para el caso de los países en desarrollo que se caracterizan por la falta de mercados integrales de seguros y créditos, la existencia de instituciones financieras formales débiles, la inestabilidad en el ingreso y la falta de seguridad social para el retiro 
(Rosenzweig, 2001). A ello se agrega la presencia de cambios socioeconómicos y demográficos acelerados, en los que adquiere relevancia el efecto de la estructura del hogar y el impacto diferenciado de la dependencia debido a los jóvenes y a las personas en edades avanzadas (Thi et al., 2013). Además, prevalecen distintos patrones de ahorro; por ejemplo, existe poca evidencia de perfiles de ahorro con forma de $\mathrm{U}$ invertida, ya sea por la presencia de escaso ahorro a cualquier edad o por la mayor probabilidad de ahorro en las edades avanzadas (Deaton, 1997). Por ello, las investigaciones empíricas recientes han ampliado el marco explicativo del ahorro y, con el uso de datos microeconómicos, se ha agregado al análisis el efecto de factores sociales, demográficos, económicos, sociológicos e institucionales (Butelmann y Gallego, 2001; Chowa, Masa y Ansong, 2012).

En el caso de México, se ha dedicado particular atención al análisis del comportamiento del ahorro de los hogares y su relación con el sexo, el nivel de escolaridad y el acceso a la seguridad social del jefe del hogar (Nava, Brown y Domínguez, 2014; Solís y Villagómez, 1999; Attanasio y Székely, 1999), y la presencia y estructura de edad de los hijos (Montes y Villagómez, 2002) y de las personas mayores en el hogar (Ceballos 2015).

Además, se resalta la importancia del ingreso. En un análisis descriptivo sobre el monto y distribución del ahorro, Székely (1998) señala que las decisiones de transferir consumo al futuro se concentran en los tres deciles de la población de mayor ingreso. Attanasio y Székely (1999) destacan el efecto del nivel superior de escolaridad, proxy del ingreso permanente, sobre el ahorro. Fuentes y Villagómez (2001) analizan el comportamiento del ahorro de los hogares de bajos ingresos a partir de los siguientes criterios: localidad rural, ausencia de ingresos financieros y percentil de ingresos. Bernal (2007) se enfoca en el estudio de los hogares pobres; esto es, aquellos por debajo del umbral oficial de pobreza. Ambos estudios encuentran evidencia de la capacidad de ahorro entre los hogares considerados pobres, con particularidades como menores montos y frecuencias, distintos instrumentos y diferentes motivaciones.

Con base en estos antecedentes, se plantea la necesidad de ampliar la investigación sobre el comportamiento del ahorro a nivel microeconómico. A diferencia de los estudios antes referidos, en este artículo se analizan los impactos marginales de las variables sociodemográficas y económicas a lo largo de toda la distribución del ahorro, de esta forma se tiene información de los hogares con altas y bajas tasas de ahorro. 


\section{LA ENIGH Y LA ESTIMACIÓN DEL AHORRO}

En este documento se utilizan los microdatos de la ENIGH 2014 en su versión tradicional. Ésta es una encuesta que levanta bienalmente el Instituto Nacional de Estadística y Geografía (INEGI) para proporcionar información estadística sobre el comportamiento de los ingresos y gastos de los hogares, en relación con su monto, procedencia y distribución. Además, ofrece información sobre las características sociodemográficas y ocupacionales de las personas y el equipamiento del hogar y la infraestructura de la vivienda.

La ENIGH tiene representatividad a nivel nacional para las áreas urbana y rural. ${ }^{1}$ Es una encuesta probabilística con diseño estratificado, bietápico y por conglomerados, en la que la unidad última de selección es la vivienda y la unidad de observación es el hogar. El tamaño de la muestra es de 19,479 hogares y la muestra expandida corresponde a 31'671,002 hogares. En este trabajo la unidad de análisis son los hogares, debido a que la mayor parte de la información sobre gasto en consumo que proporciona la encuesta está disponible a nivel de los hogares.

Esta fuente de información es el instrumento más especializado para analizar el ahorro de los hogares debido a su diseño y contenido. De hecho, la mayoría de las investigaciones para el caso de México la utilizan. ${ }^{2}$ En el 2014 el INEGI presentó por última vez los resultados en dos versiones: nueva construcción y tradicional. ${ }^{3}$

En relación con la medición del ahorro y siguiendo a Székely (1998), éste se estima como la resta del gasto en consumo corriente que realizan los integrantes del hogar del ingreso corriente declarado. ${ }^{4}$ Incluye componentes monetarios y no monetarios. La tasa de ahorro se obtiene de dividir el ahorro por el ingreso corriente. Enseguida se presentan las estimaciones del ahorro para los hogares en México.

\footnotetext{
1 Además del estado de Tabasco, que en su momento acordó con el INEGI una ampliación de la muestra.

2 Algunas excepciones son Bernal (2007) y Ceballos (2015). Ambos autores utilizan la Encuesta Nacional sobre Niveles de Vida de los Hogares, proyecto conjunto de la Universidad Iberoamericana, el Centro de Investigación y Docencia Económicas y la participación de la Universidad de Duke en los Estados Unidos. El resto de documentos citados para el caso de México toman como referencia la ENIGH (Attanasio y Székely, 1999; Solís y Villagómez, 1999; Fuentes y Villagómez, 2001; Montes y Villagómez, 2002; Nava, Brown y Domínguez, 2014; Valles y Aguilar, 2015).

3 A partir de 2008, el INEGI publica los resultados de la ENIGH en la versión nueva construcción, que incorpora las recomendaciones de la Organización de las Naciones Unidas y que sirve de base al Módulo de Condiciones Socioeconómicas (MCS), anexo a la ENIGH. El MCS es el instrumento que utiliza el Consejo Nacional para la Evaluación de la Política de Desarrollo Social para la medición de la pobreza multidimensional. Con el objetivo de garantizar la comparabilidad de la encuesta con los años previos, el INEGI continuó con la publicación de la versión tradicional (INEGI, 2009). En 2016, el INEGI levantó una nueva serie de la ENIGH, en la que la construcción de las variables es distinta a la versión tradicional de la encuesta (INEGI, 2017).

4 El ingreso corriente cumple con los criterios de regularidad y disponibilidad, y excluye a los flujos que modifican el patrimonio neto del hogar (INEGI, 2009).
} 


\section{EL AHORRO DE LOS HOGARES MEXICANOS}

En la revisión del ahorro de los hogares, una de las primeras interrogantes que aparecen es: ¿Cuánto ahorran los hogares mexicanos? De acuerdo con los microdatos de la ENIGH 2014, el ahorro medio como proporción del ingreso medio total de los 31.6 millones de hogares fue de 12.9 por ciento, mientras que el promedio de la tasa de ahorro fue -1.7 por ciento, debido a la presencia de hogares con ahorro negativo. ${ }^{5}$

Llama la atención la gran diferencia en el comportamiento del ahorro según deciles de ingreso de los hogares. La gráfica 1 ilustra la tasa de ahorro por deciles. En ella se observa que los hogares de los deciles I a IV registran tasas negativas; se trata de unidades que son deudoras netas. Es posible que estén haciendo uso de recursos previamente acumulados para hacer frente a una reducción imprevista en el ingreso. Los hogares del decil $\mathrm{V}$ registran tasas de ahorro cercanas a cero (0.17 por ciento); es decir, tienen una baja capacidad de ahorro. En el decil IX la tasa fue 17.7 por ciento y en el $\mathrm{X}$ fue 26.3 por ciento. Al comparar estos resultados con los de Attanasio y Székely (1999) se aprecia que las desigualdades en la capacidad de ahorro se mantienen en el tiempo.

Estas cifras visibilizan la presencia de "diferencias radicales entre los agentes económicos según su posición en la distribución” (Székely, 1998: 293). Como señala el autor, la mayoría de las investigaciones que analizan los factores explicativos del ahorro parten del supuesto de un "agente económico representativo", condición que resulta restrictiva.

Una vez analizadas las tasas de ahorro de los hogares mexicanos, el objetivo de las siguientes secciones es estudiar los factores explicativos del ahorro: no sólo los económicos (ingreso, educación, acceso a mercados financieros y redes sociales), sino también los sociodemográficos (sexo y edad del jefe de hogar, clase de hogar, y presencia de menores y mayores en el hogar).

\footnotetext{
Estos resultados coinciden con los hallazgos de Attanasio y Székely (1999). Los autores estiman que entre 1984 y 1996 las tasas de ahorro oscilaron entre 8.5 y 14.1 por ciento y las tasas medias registraron valores negativos, con excepción del año 1994.
} 
Gráfica 1. Tasa de ahorro por deciles de los hogares (porcentaje del ingreso corriente).

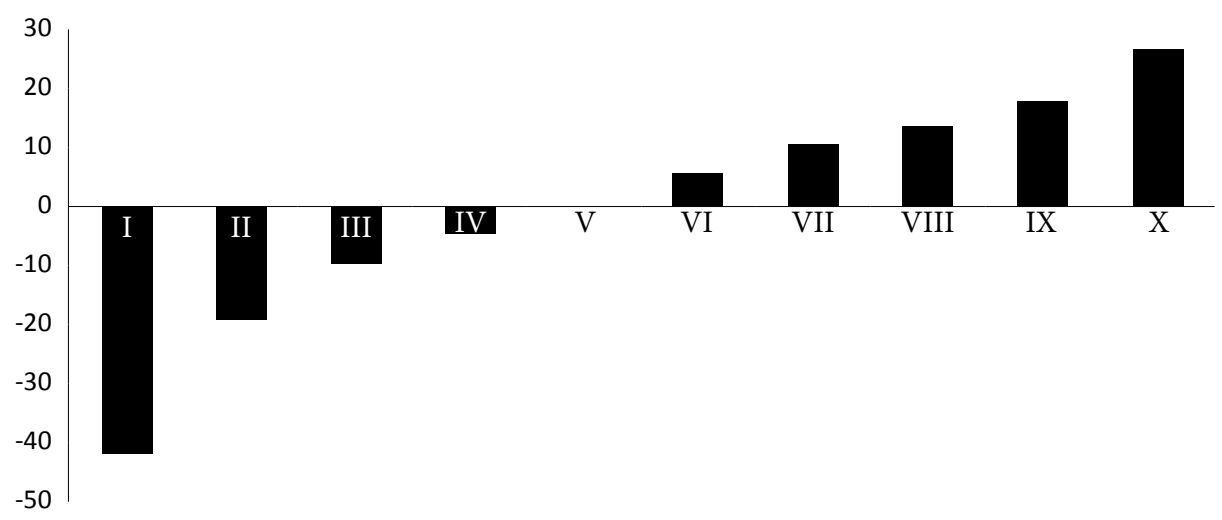

Fuente: Elaboración propia con base en microdatos de la ENIGH 2014, INEGI.

\section{III.1. Factores económicos}

Ingreso. La relación entre el ingreso -transitorio y permanente- y el ahorro ha sido analizada para países desarrollados (Browning y Lusardi, 1996; Bosworth et al., 1991; Poterba, 1994; Dynan, Skinner y Zeldes, 2004) y para países en desarrollo (Denizer y Wolf, 1998; Coronado, 1998; Attanasio y Székely, 1999; Székely, 1998). Los resultados muestran consenso en cuanto a la relación positiva entre ambas variables. Sin embargo, no lo hay en torno al tipo de ingreso que se incluye como factor explicativo, debido, en parte, a las dificultades para medir tanto el ingreso a lo largo del ciclo de vida, como el ingreso transitorio y permanente. En este trabajo se analiza el efecto del ingreso sobre el ahorro con base en la HIP.

A fin de ilustrar las diferencias en el ingreso, se organizan los hogares según cuantiles de ahorro. Como era de esperarse, aparecen desigualdades significativas. El ingreso promedio en Q20 es superior a los ocho mil pesos mensuales, mientras que en Q50 está por encima de los 11 mil pesos y en Q90 sobrepasa los 17 mil pesos.

Educación. Algunos autores (Butelmann y Gallego, 2000; Dynan et al., 2004) incluyen la variable "educación" como proxy del ingreso permanente. Otros (Székely, 1998), la incorporan como indicador del acervo de capital humano. En relación con el signo de la variable, la mayoría de los estudios encuentran una relación positiva entre los niveles de educación y las tasas de ahorro de los hogares. La relación positiva se atribuye, por un lado, a la influencia que pudiera tener la educación en el ingreso y, posteriormente, el ingreso en la tasa de ahorro 
(Attanasio, 1998). Por el otro lado, existen algunas explicaciones que relacionan directamente el nivel educacional y la tasa de ahorro. Por ejemplo, las personas que invierten más en educación presentan una tasa de descuento menor, que a su vez los lleva a incrementar el ahorro (Browning y Lusardi, 1996). Respecto a la relación negativa, ésta se explica por un cambio en las preferencias hacia el consumo de bienes más costosos (Burney y Khan, 1992 citado por Székely, 1998). También, es posible que los jefes de hogar con un alto nivel de educación prevean una tendencia ascendente del ingreso a lo largo de la vida, lo que impulsaría el consumo y deprimiría el ahorro (Bebczuk et al., 2015).

La estadística descriptiva muestra que en los hogares donde el jefe de hogar tiene estudios de licenciatura y más, la tasa de ahorro es de 16.6 por ciento; mientras que en los hogares donde el jefe de hogar tiene estudios de primaria, es de 11.8 por ciento. Sin embargo, estos resultados deben tomarse con reserva, ya que los porcentajes no se controlan por el ingreso y existe evidencia de una alta correlación entre ambas variables (cuadro 1).

Acceso a mercados financieros. El acceso efectivo a los mercados financieros tiene un impacto relevante en las asignaciones de consumo y en las decisiones de inversión; en consecuencia, también lo tienen en el ahorro (Butelmann y Gallego 2000, 2001). Las tasas de ahorro que aparecen en el cuadro 1 indican que ésta es significativamente mayor en los hogares que reportaron gastos financieros relacionados con depósitos de ahorro, uso de tarjetas de crédito y compras de valores, entre otros gastos financieros y de capital: 16.1 por ciento en comparación con 8.2 por ciento en los hogares sin acceso a mercados financieros.

Acceso a redes de transferencias. El acceso a redes de transferencias económicas, ya sea de carácter formal o informal, facilita la obtención de recursos de los hogares, sobre todo en situaciones de emergencia e imprevistos (Butelmann y Gallego, 2000,2001). De aquí que la tasa de ahorro sea mayor cuando hay acceso a ellas. Las estadísticas del cuadro 1 indican que la tasa de ahorro en los hogares con redes de transferencias es 15.7 por ciento y en los hogares sin acceso es de 10.9 por ciento. 
Cuadro 1. Ahorro de los hogares según características económicas.

(Porcentaje del ingreso corriente total).

\begin{tabular}{lcc}
\hline & Subgrupos & Tasas de ahorro \\
\hline Ninguna & Educación & \\
Primaria & & 11.2 \\
Secundaria & 11.8 \\
Preparatoria & 10.3 \\
Licenciatura y más & 10.7 \\
\hline & & 16.6 \\
\hline Ausencia & Mercados financieros & \\
Presencia & & 8.2 \\
& & 16.1 \\
\hline Ausencia & Redes de transferencias & 10.9 \\
Presencia & 15.7 \\
\hline
\end{tabular}

Fuente: Elaboración propia con base en microdatos de la ENIGH 2014, INEGI.

\section{III.2. Factores sociodemográficos}

Sexo del jefe. Distintos autores encuentran evidencia de menores tasas de ahorro en los hogares de jefatura femenina, en comparación con las jefaturas masculinas (Nava, Brown y Domínguez, 2014). Las desigualdades de género se atribuyen a que las mujeres dedican una mayor proporción del ingreso familiar al consumo de bienes y servicios de capital humano (salud y educación) y de esparcimiento (Hira y Loibl, 2008). Duncan (1993) encuentra que es más probable que las mujeres manejen las tareas de rutina de administración del dinero. Además, cuando las mujeres invierten, prefieren inversiones menos riesgosas y toman las decisiones con sus cónyuges, más que de manera independiente. Por el contrario, Seguino y Floro (2003) plantean que las responsabilidades asociadas a las mujeres resultan en un ahorro elevado por motivos de precaución debido a la necesidad, desde su percepción, de suavizar el consumo familiar frente al de los hombres. Las cifras del cuadro 2 muestran que los hogares dirigidos por mujeres registran tasas de ahorro significativamente menores (9.4 por ciento), en comparación con los hombres (14.0 por ciento).

Edad del jefe. En relación con los aspectos demográficos, otra de las variables más analizadas es la edad. En el marco de la HCV, los estudios anticipan un perfil de U invertida. Sin embargo, Deaton (1992) encuentra evidencia de que los hogares dirigidos por personas mayores ahorran o al menos no desahorran tanto 
como predice la HCV. El autor lo atribuye a un posible efecto de selectividad por edad. También, es posible que en las edades más avanzadas aumenten los incentivos a ahorrar frente a los mayores riesgos de enfermedades crónico-degenerativas e incapacitantes (De Nardi, French y Jones, 2010) o el mayor interés por dejar una herencia o legado (Hurd, 1987).

Para el caso de México, la menor tasa de ahorro aparece en el tramo de edades menores de 30 años y el porcentaje más alto corresponde al grupo de edad 50-59, momento a partir del cual disminuye. Resaltan las altas tasas en las edades más avanzadas, en comparación con las edades intermedias o laborales (cuadro 2).

Clase de hogar. La relación entre los distintos tipos de hogar y el ahorro se explica a partir del aprovechamiento de las economías de escala, sobre todo en el caso particular de los hogares ampliados (Bernal, 2007). Se observa que las tasas de ahorro más altas corresponden a las familias ampliadas (14.8 por ciento), mientras que las menores corresponden a los hogares unipersonales (cuadro 2).

Integrantes menores (0-11 años). La evidencia empírica para el caso mexicano muestra que la presencia de infantes en los hogares reduce el ahorro (Montes y Villagómez, 2002). Sin embargo, el comportamiento del ahorro puede estar más ligado a las edades de los infantes, que a la presencia o número (Espenshade, 1975). Las cifras que aparecen en el cuadro 2 muestran que la tasa de ahorro de los hogares sin integrantes menores ( 0 a 11 años) es 15.2 por ciento, en contraste con la tasa del resto de los hogares, que es 10.3 por ciento.

Integrantes mayores (65+). El efecto de esta variable es discrepante en los estudios. Ceballos (2015) encuentra que en los hogares mexicanos dirigidos por personas menores a 50 años la presencia de personas mayores se relaciona con mayor ahorro monetario, pero una vez que el jefe de hogar alcanza esa edad, la tendencia se revierte y son los hogares sin personas mayores los que presentan mayor ahorro. En este caso, las tasas más elevadas de ahorro corresponden a los hogares sin personas mayores (cuadro 2).

Localidad de residencia. Los resultados muestran que el ahorro de los hogares en zonas rurales es menor al que logran los hogares urbanos; una explicación es que en estos últimos los servicios suelen estar más articulados y consolidados (Valles y Aguilar, 2015). Además, los mecanismos de ahorro son diferenciados según localidad; en las zonas rurales son más comunes los instrumentos informales (Minh et al., 2013). Para el caso de los hogares analizados, el cuadro 2 muestra que la tasa de ahorro de los hogares urbanos es marcadamente mayor. 
Cuadro 2. Ahorro de los hogares según características sociodemográficas.

(Porcentaje del ingreso corriente total).

\begin{tabular}{|c|c|}
\hline Subgrupos & Tasas de ahorro \\
\hline \multicolumn{2}{|c|}{ Sexo del jefe } \\
\hline Masculino & 14.0 \\
\hline Femenino & 9.4 \\
\hline \multicolumn{2}{|c|}{ Edad del jefe } \\
\hline Menores de 30 & 4.1 \\
\hline $30-39$ & 9.4 \\
\hline $40-49$ & 8.8 \\
\hline $50-59$ & 18.8 \\
\hline $60+$ & 17.4 \\
\hline \multicolumn{2}{|c|}{ Clase de hogar } \\
\hline Nuclear & 12.9 \\
\hline Ampliado o compuesto & 14.8 \\
\hline Unipersonal o corresidente & 6.7 \\
\hline \multicolumn{2}{|c|}{ Integrantes menores ( 0 a 11 años) } \\
\hline Presencia & 10.3 \\
\hline Ausencia & 15.2 \\
\hline \multicolumn{2}{|c|}{ Integrantes mayores $(65+)$} \\
\hline Presencia & 12.5 \\
\hline Ausencia & 14.7 \\
\hline \multicolumn{2}{|c|}{ Localidad de residencia } \\
\hline Rural & 8.7 \\
\hline Urbana & 13.5 \\
\hline
\end{tabular}

Fuente: Elaboración propia con base en microdatos de la ENIGH 2014, INEGI.

\section{ANÁLISIS DE REGRESIÓN CUANTÍLICA DEL AHORRO}

La estimación de un modelo econométrico para analizar los determinantes del ahorro enfrenta dos problemas. Primero, un posible sesgo de selección derivado de que el grupo de hogares ahorradores tiene características específicas que determinan su autoselección en este grupo. Segundo, una posible endogeneidad de los ingresos en la ecuación de ahorro.

Las técnicas más utilizadas para evitar estos problemas son las estimaciones de modelos de dos etapas (Bernal, 2007) y modelos tipo Tobit (Tobin,1958), (Nava, Brown y Domínguez, 2014; Valles y Aguilar, 2015). Una cuestión adicional se refiere a la posibilidad de que los factores explicativos difieran a lo largo de la distribución del ahorro. En este tema son relativamente pocos los estudios que analizan la heterogeneidad de los determinantes del ahorro utilizando la regresión cuantílica (Chen, Kuan y Lin, 2007). 
Sin embargo, los modelos de regresión cuantílica no resuelven el posible problema de la endogeneidad que se produce al incluir el ingreso en la función del ahorro. Una propuesta reciente que permite solucionar esta limitación es la estimación cuantílica con variables instrumentales, método desarrollado por Chernozhukov, Fernandez-Val y Kowalski (2015). Los autores señalan que los coeficientes considerados bajo esta metodología son consistentes y estiman sus intervalos de confianza, lo que permite comparar los resultados con los que arroja la regresión cuantílica.

Con base en estos elementos, el punto de partida fue la estimación de la regresión por cuantiles, en la que se incluyó como variable explicativa la estimación del ingreso permanente a partir de la especificación que se describe más adelante. Posteriormente, para abordar el problema de la endogeneidad del ingreso, se estimó la regresión cuantílica con variables instrumentales, considerando la misma ecuación propuesta para la regresión por cuantiles como ecuación estructural. Para la ecuación reducida se incluyeron como instrumentos la escolaridad y el ingreso derivado de fuentes distintas al trabajo. Al comparar las dos estimaciones, fue posible reflexionar en torno a los posibles sesgos que provienen del problema de la endogeneidad.

\section{IV.1. Especificación del modelo}

La especificación funcional del modelo a estimar es la siguiente:

$$
\begin{gathered}
s_{i}=\alpha_{o}^{\theta}+\alpha_{1}^{\theta} \operatorname{Ing} \operatorname{Per}_{l}+\beta^{\theta} X_{1 i}+\beta^{\theta} X_{2 i}+\varepsilon_{o}^{\theta} \\
i=1,2, \ldots n
\end{gathered}
$$

Donde $i$ representa los índices de los hogares, $\theta$ los índices de los cuantiles, $s_{i}$ es la tasa de ahorro para cada hogar $i$, Ing Per es el ingreso permanente estimado, $X_{1}$ representa un vector de características económicas de los hogares, $X_{2}$ es un vector de características sociodemográficas del jefe de hogar y de los hogares, el coeficiente $\alpha_{1}$ mide la propensión marginal a ahorrar fuera del ingreso permanente y $\varepsilon_{1}$ es el término de perturbación.

En relación con la variable dependiente $\left(s_{i}\right)$, ésta incluye valores negativos y positivos, evitando así el problema de censura. Además, como señala Sheiner, 1995 (citado por Chen, Kuan y Lin, 2007), se parte de que los hogares con ahorro negativo pueden contener tanta información sobre la acumulación de activos como aquella que proporcionan los hogares con ahorros positivos. Para evitar el problema del ahorro negativo indefinido en el logaritmo, $s_{i}$ se obtiene 
como $(\ln Y-\ln C)$. Recordemos que, para valores pequeños, la tasa de ahorro puede aproximarse como la diferencia entre los logaritmos de ingreso y consumo (Deaton y Paxson, 1994, citado por Chen, Kuan y Lin, 2007).

El cuadro 3 incluye la operacionalización de las variables explicativas agrupadas en factores económicos y sociodemográficos. En la última columna aparece el resultado de la revisión bibliográfica y empírica previa, el efecto esperado de las variables independientes sobre la tasa de ahorro y la tendencia intercuantil esperada.

Cuadro 3. Operacionalización de las variables explicativas y efecto esperado.

\begin{tabular}{|c|c|c|}
\hline Variable & Operacionalización & $\begin{array}{l}\text { Signo esperado / tendencia } \\
\text { intercuantil esperada }\end{array}$ \\
\hline \multicolumn{3}{|c|}{ Factores económicos } \\
\hline Ingreso permanente & Estimada & + / creciente \\
\hline Mercados financieros & $1=$ acceso, $0=\sin$ acceso & + / creciente \\
\hline Redes de transferencias & $1=$ acceso, $0=\sin$ acceso & + / decreciente \\
\hline Personas ocupadas & $\begin{array}{l}\text { Número de personas que tienen trabajo } \\
\text { y } 14 \text { o más años de edad }\end{array}$ & + / creciente \\
\hline \multicolumn{3}{|c|}{ Factores sociodemográficos } \\
\hline Sexo del jefe & $1=$ mujer 0 =hombre & - / decreciente \\
\hline Edad del jefe & Número de años cumplidos & + / creciente \\
\hline Edad del jefe al cuadrado & & - / creciente \\
\hline Clase de hogar & $\begin{array}{l}\text { 1=nuclear (categoría de referencia) } \\
2=\text { ampliado o compuesto } \\
3=\text { unipersonal }\end{array}$ & $\begin{array}{l}\text { +/ creciente } \\
\text { - / decreciente }\end{array}$ \\
\hline Número de menores & $\begin{array}{l}\text { Número de integrantes que tienen } 11 \text { o menos } \\
\text { años de edad }\end{array}$ & - / decreciente \\
\hline Número de mayores & $\begin{array}{l}\text { Número de integrantes que tienen } 65 \text { o más años } \\
\text { de edad }\end{array}$ & - / decreciente \\
\hline Localidad de residencia & $1=$ urbana, $0=$ rural & + / creciente \\
\hline
\end{tabular}

En virtud de que el ingreso permanente no es directamente observable, se optó por realizar su estimación e incluir la variable estimada en la función de la tasa de ahorro. La mayor parte de los estudios utilizan distintos métodos econométricos para su cálculo. Algunos sólo tienen la intención de estimar al ingreso permanente (Hall, 1978; Campbell y Mankiw, 1990; Flavin, 1981; Manitsaris, 2006; Khan y Nishat, 2010; Blinder y Deaton 1985) y otros la incorporan en la función del ahorro como variable independiente (Chen, Kuan y Lin, 2007). Siguiendo la 
propuesta de Chen, Kuan y Lin (2007), el ingreso permanente se estima con una regresión de MCO con la siguiente especificación: ${ }^{6}$

$$
\operatorname{IngPer}_{i}=\alpha_{0}+\beta_{1} \operatorname{sex}_{i}+\beta_{2} e d a_{i}+\beta_{3} e d a_{i}^{2}+\beta_{3} \operatorname{esc}_{i}+\beta_{4} \text { per }_{i}+\beta_{3} i n g_{n t_{i}}+\varepsilon_{i}
$$

Donde se incluyen como factores explicativos: sexo (sex), edad (eda), edad al cuadrado $\left(e d a^{2}\right)$, escolaridad (esc), perceptores de ingreso (per) e ingreso derivado de fuentes distintas al trabajo $\left(\right.$ ing $\left._{n t}\right)$.

\section{IV.2. Resultados de la estimación}

La regresión cuantílica permitió analizar el impacto de los distintos factores económicos y sociodemográficos sobre la variable endógena, tomando en consideración la localización de cada cuantil en la distribución (QR20, QR50, QR60 y QR90). Al minimizar las desviaciones absolutas ponderadas con pesos asimétricos, la estimación con valores atípicos no se ve alterada por valores extremos, ya que "penaliza" los errores de forma lineal. Por ejemplo, para QR20 los residuos positivos se ponderan con 0.20 , mientras que los negativos con 0.80 (Vicéns y Sánchez, 2012).

Los parámetros estimados $\left(\beta_{\theta}\right)$ representan, al igual que en MCO, los efectos marginales de las variables independientes sobre la tasa de ahorro, pero con la diferencia de que es posible calcular distintos parámetros $\left(\beta_{\theta}\right)$ que pueden variar entre cuantiles. Así, las discrepancias de los efectos marginales entre cuantiles muestran la heterogeneidad entre los diferentes grupos de hogares. Cabe señalar que la estimación se realizó con el método de errores estándar robustos para evitar problemas de heterocedasticidad.

En el cuadro 4 se presentan los resultados de las distintas estimaciones con MCO y para QR20, QR50, QR60 y QR90. En primer lugar, se confirma el efecto positivo del ingreso permanente sobre la tasa de ahorro. Como se observa, en promedio la propensión marginal al ahorro es de 0.09 estimada con MCO; ello significa que un aumento del 10 por ciento en el ingreso permanente eleva la tasa del ahorro en 0.9 por ciento en promedio. Tal como se esperaba, la propensión marginal al ahorro estimada es menor en el cuantil inferior (0.06) y en el superior se registra la más elevada (0.16). Ello se demuestra además con la prueba de

\footnotetext{
6 Los resultados aparecen en el cuadro 1 del anexo.
} 


\section{las diferencias entre cuantiles ${ }^{7}$ que resultó estadísticamente significativa y con} signo positivo (cuadro 5). En la misma dirección, la gráfica 2 ilustra la heterogeneidad de la propensión marginal al ahorro entre cuantiles. ${ }^{8}$ Estos resultados coinciden con los de Chen, Kuan y Lin (2007).

\section{Gráfica 2. Propensión marginal al ahorro entre cuantiles.*}

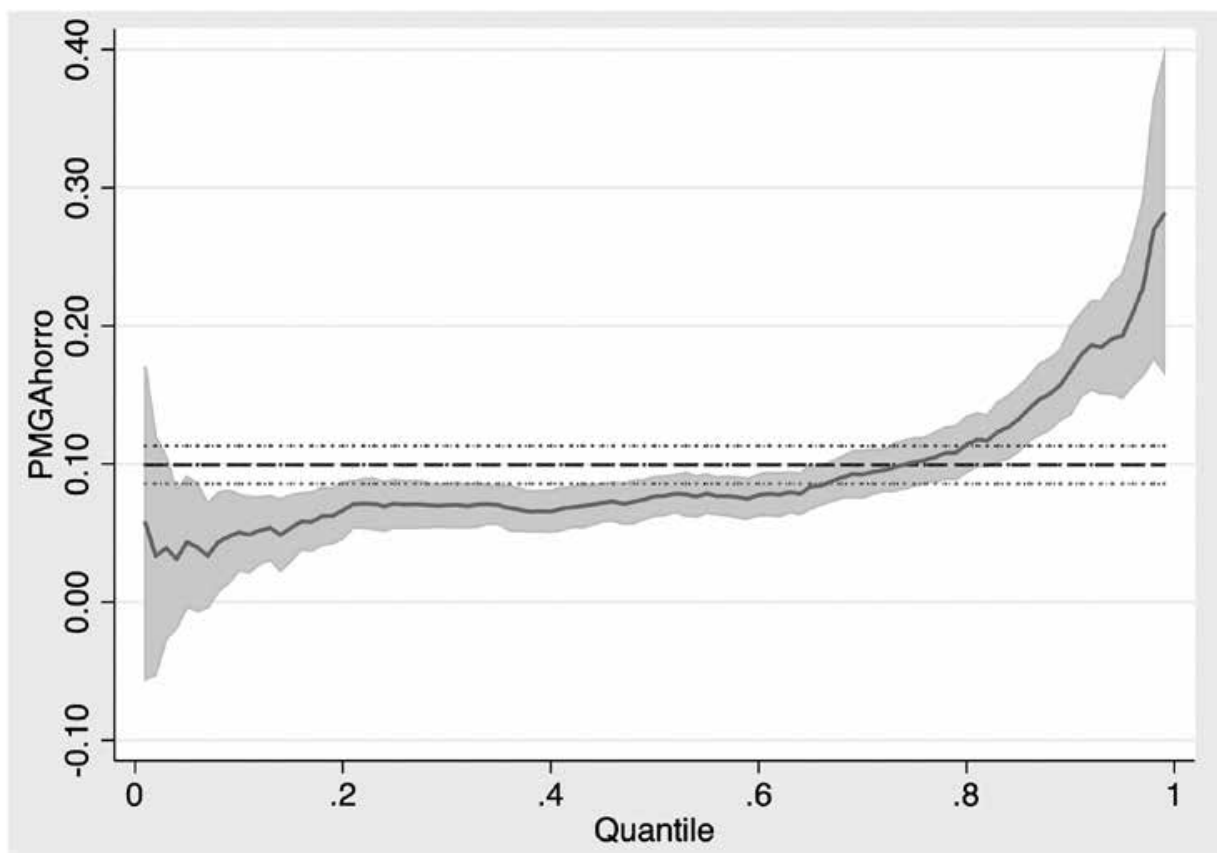

Fuente: Estimaciones propias con base en microdatos de la enigh 2014, INEGI.

*Coeficiente del ingreso permanente.

7 El resultado de estas estimaciones se interpreta como el cuantil superior menos el inferior; por tanto, el signo positivo implica un ascenso en el comportamiento del coeficiente entre los dos cuantiles y viceversa, un signo negativo implica un comportamiento descendiente.

8 La heterogeneidad de la propensión marginal al ahorro entre cuantiles está representada por la línea sólida. Los contornos de la nube de puntos sombreada corresponden a los valores inferior y superior de la banda de confianza del correspondiente parámetro estimado en la regresión cuantílica. La línea horizontal con guiones largos corresponde al valor del estimador de la media condicional estimado con MCO acompañada también por los puntos de su banda de confianza. 
Se comprueba la importancia de la relación entre el resto de los factores económicos y la tasa de ahorro. El acceso a mercados financieros resultó estadísticamente significativo en todos los cuantiles, con excepción de QR20 (cuadro 4). Los coeficientes más altos son los de los cuantiles intermedios (QR50 y QR60); se confirma, a su vez, que la diferencia de los coeficientes en estos cuantiles es estadísticamente significativa con signo positivo (cuadro 5).

El coeficiente asociado al acceso a redes de transferencias fue significativo en todos los casos, con una magnitud muy similar entre ellos, por lo que la prueba de la diferencia de coeficientes no resultó estadísticamente significativa; es decir el efecto del coeficiente es similar a lo largo de la distribución. Estos resultados corroboran la relevancia que tienen los recursos financieros en las decisiones de inversión y consumo y, por tanto, en la tasa del ahorro, tal como lo muestran Butelmann y Gallego (2001).

Por último, el coeficiente asociado al número de personas ocupadas en el hogar resultó estadísticamente significativo en todos los cuantiles y también la prueba de diferencias entre ellos con signo positivo. Como era de esperarse, la presencia de economías de escala que proceden del mayor número de integrantes capaces de aportar un ingreso liberan recursos para el ahorro.

En los factores sociodemográficos, el signo asociado al sexo de la jefatura femenina resultó negativo en todos los cuantiles con excepción de QR20 en el que no fue significativo (cuadro 4); por lo que la prueba de diferencias intercuantil resultó significativa entre QR20 y QR90, pero no en los intermedios en donde el coeficiente es similar (cuadro 5). Ello confirma las desigualdades por sexo y las condiciones de desventaja que enfrentan las jefas de hogar (Nava, Brown y Domínguez, 2014).

En relación con la edad del jefe, no se encontró una asociación significativa. Una explicación posible es que, al ser un análisis de corte transversal, puede ser inadecuado para caracterizar el ahorro a lo largo del ciclo vital.

Tampoco se comprueba el efecto de la clase de hogar sobre la tasa de ahorro. Cabe mencionar que ésta es una variable poco analizada en la bibliografía. Si bien esperábamos que las familias ampliadas tuvieran un efecto positivo, al controlar por el número de personas el efecto no fue estadísticamente significativo.

El número de menores en el hogar incide de manera negativa sobre la tasa de ahorro. El coeficiente asociado a los menores de edad es negativo en todos los cuantiles (cuadro 4). Las diferencias intercuantiles de los coeficientes son estadísticamente significativas y, como era de esperarse, con signo negativo (cuadro 5). Este resultado coincide con los hallazgos de otras investigaciones (Montes y Villagómez, 2002), en el sentido de que la presencia de infantes en el hogar provoca una caída del ahorro, ya que se modifican los patrones de consumo e ingreso de los hogares. 
El número de mayores en el hogar sólo resultó estadísticamente significativos en QR20 y QR50 (cuadro 4); por tanto la prueba intercuantil no es estadísticamente significativa (cuadro 5). Así, la hipótesis de que las personas en edades avanzadas ahorran más por precaución (Butelmann y Gallego, 2001) o porque aportan ingresos al hogar (Valles y Aguilar, 2015) se confirma parcialmente.

El coeficiente asociado a la localidad urbana resultó estadísticamente significativo en todos los casos. Sin embargo, el coeficiente disminuye para los cuantiles superiores, incluso en Q90 es negativo (cuadro 4). La reducción del coeficiente a lo largo de la distribución coincide con los hallazgos de Chen, Kuan y Lin (2007). Es posible que entre los cuantiles más altos las oportunidades que ofrecen las economías de las localidades urbanas sean menos importantes para tener un excedente que ahorrar. La prueba intercuantil también resultó significativa y con signo negativo (cuadro 5).

Cuadro 4. Resultados de la estimación por mco y regresión cuantílica (QR20, QR50, QR60 y QR90).

\begin{tabular}{|c|c|c|c|c|c|}
\hline Variables & MCO & QR_20 & QR_50 & QR_60 & QR_90 \\
\hline Ingreso permanente & $0.090 * * *$ & $0.057 * * *$ & $0.066 * * *$ & $0.071^{* * *}$ & $0.161 * * *$ \\
\hline Mercados financieros & $0.022 * * *$ & 0.004 & $0.044^{* * *}$ & $0.057 * * *$ & $0.043 * * *$ \\
\hline Redes de transferencias & $0.026^{* * *}$ & $0.021 * *$ & $0.025^{* * *}$ & $0.019 * *$ & $0.028^{* *}$ \\
\hline Personas ocupadas & $0.076 * * *$ & $0.059 * * *$ & $0.065^{* * *}$ & $0.066 * * *$ & $0.091 * * *$ \\
\hline Sexo del jefe & $-0.016^{* *}$ & -0.001 & $-0.020 * * *$ & $-0.027^{* * *}$ & $-0.037 * * *$ \\
\hline Edad del jefe & $-0.003 * * *$ & -0.001 & 0.001 & -0.001 & $-0.006 * * *$ \\
\hline Edad del jefe & $0.000 * * *$ & $0.000 * *$ & 0.000 & $0.000^{* * *}$ & $0.000 * * *$ \\
\hline Hogar ampliado & 0.007 & 0.015 & -0.005 & 0.007 & 0.015 \\
\hline Hogar unipersonal & 0.011 & $0.048 * * *$ & -0.001 & -0.010 & 0.014 \\
\hline Número de menores & $-0.017 * * *$ & $-0.008^{* * *}$ & $-0.013^{* * *}$ & $-0.014^{* * *}$ & $-0.025 * * *$ \\
\hline Número de mayores & 0.018 & $0.019 * *$ & $0.018^{* *}$ & 0.005 & -0.002 \\
\hline Localidad urbana & $0.028 * * *$ & $0.063^{* * *}$ & $0.018^{* * *}$ & $0.015^{* * *}$ & $-0.030 * * *$ \\
\hline Constante & $-0.976 * * *$ & $-0.962 * * *$ & $-0.787^{* * *}$ & $-0.725^{* * *}$ & -1.154 \\
\hline $\mathrm{N}$ & 18,191 & 18,191 & 18,191 & 18,191 & 18,191 \\
\hline Fvalue & 0.00 & & & & \\
\hline R-squared & 0.07 & 0.024 & 0.035 & 0.040 & 0.052 \\
\hline
\end{tabular}

Fuente: Estimaciones propias con base en microdatos de la ENIGH 2014, INEGI.

Nota: Estadísticamente significativo al $0.01^{* * *}, 0.05^{* *}$ y $0.10^{*}$. La estimación pondera a los hogares por el inverso de su probabilidad de ser incluidos dado el número de hogares que representa en la encuesta $\left(\right.$ fesc $=\frac{n}{N}$. factor $)$. 
Cuadro 5. Resultados de los rangos intercuantil.

\begin{tabular}{lrr}
\hline \multicolumn{1}{c}{ Variables } & Q20-Q90 & \multicolumn{2}{c}{ Q50-Q90 } \\
\hline Ingreso permanente & $0.11^{* * *}$ & $0.10^{* * *}$ \\
Mercados financieros & $0.03^{* * *}$ & -0.01 \\
Redes de transferencias & 0.01 & 0.02 \\
Personas ocupadas & $0.03^{* * *}$ & $0.02^{* * *}$ \\
Sexo del jefe & $-0.02^{* * *}$ & 0.00 \\
Número de menores & $-0.01^{* *}$ & $-0.01^{* * *}$ \\
Número de mayores & -0.01 & -0.01 \\
Localidad urbana & $-0.08^{* * *}$ & $-0.04^{* * *}$ \\
\hline
\end{tabular}

Fuente: Estimaciones propias con base en microdatos de la ENIGH 2014, INEGI.

\section{IV.3. Estimación cuantílica con variables instrumentales}

Las investigaciones que se aproximan al estudio del ahorro a través de estimaciones cuantílicas han dedicado poca atención a la endogeniedad, quizá por la ausencia de métodos econométricos adecuados para esta finalidad. Chernozhukov, Fernandez-Val y Kowalski (2015) proponen una metodología para abordar este problema a través de estimaciones cuantílicas con variables instrumentales.

En el cuadro 6 se presentan los intervalos de confianza al 95 por ciento (superior e inferior) calculados con este método. La ventaja de esta estimación es el cálculo de los intervalos de confianza, tomando en consideración la endogeneidad del ingreso. En un primer ejercicio comparativo de los coeficientes QR con aquellos obtenidos con variables instrumentales, no fue posible encontrar un patrón claro en cuanto a las diferencias en la magnitud de los coeficientes estimados con uno u otro método. Por lo tanto, sólo se revisó si los coeficientes de estimación cuantílica arriba descritos se encontraban dentro del intervalo, como proponen Chernozhukov, Fernandez-Val y Kowalski (2015). Si bien fue imposible cuantificar la magnitud del sesgo en los coeficientes, se sugiere que el análisis realizado anteriormente con $\mathrm{QR}$ es satisfactorio debido a que sus coeficientes están dentro del intervalo de confianza. Dejamos para futuras investigaciones el avance en las rutinas y métodos para la estimación cuantílica con instrumentos, ya que ésta es sólo una primera aproximación. 
Cuadro 6. Resultados de la estimación cuantílica con variables instrumentales. *

\begin{tabular}{|c|c|c|c|c|}
\hline Variables & QR20 & QR50 & QR60 & QR90 \\
\hline \multicolumn{5}{|l|}{ Ingreso permanente } \\
\hline Inferior & -0.117 & -0.006 & 0.021 & 0.123 \\
\hline Superior & 0.086 & 0.092 & 0.090 & 0.239 \\
\hline QR & 0.057 & 0.066 & 0.071 & 0.161 \\
\hline \multicolumn{5}{|l|}{ Mercados financieros } \\
\hline Inferior & -0.048 & 0.020 & 0.021 & -0.040 \\
\hline Superior & 0.062 & 0.075 & 0.068 & 0.018 \\
\hline QR & 0.004 & 0.044 & 0.057 & 0.043 \\
\hline \multicolumn{5}{|l|}{ Redes de transferencia } \\
\hline CQIV & 0.029 & 0.025 & 0.016 & 0.003 \\
\hline Inferior & -0.146 & -0.009 & -0.017 & -0.052 \\
\hline Superior & 0.109 & 0.066 & 0.057 & 0.039 \\
\hline QR & 0.021 & 0.025 & 0.019 & 0.028 \\
\hline \multicolumn{5}{|l|}{ Personas ocupadas } \\
\hline Inferior & 0.096 & 0.070 & 0.059 & 0.046 \\
\hline Superior & 0.047 & 0.053 & 0.046 & 0.033 \\
\hline QR & 0.166 & 0.087 & 0.078 & 0.060 \\
\hline \multicolumn{5}{|l|}{ Sexo del jefe } \\
\hline Inferior & -0.063 & -0.053 & -0.061 & -0.063 \\
\hline Superior & 0.097 & 0.009 & 0.008 & 0.033 \\
\hline QR & -0.001 & -0.020 & -0.027 & -0.037 \\
\hline \multicolumn{5}{|l|}{ Edad del jefe } \\
\hline Inferior & -0.005 & 0.001 & -0.001 & -0.006 \\
\hline Superior & 0.027 & 0.009 & 0.008 & 0.006 \\
\hline QR & -0.001 & 0.001 & -0.001 & -0.006 \\
\hline \multicolumn{5}{|l|}{ Edad del jefe } \\
\hline Inferior & 0.000 & 0.000 & 0.000 & 0.000 \\
\hline Superior & 0.000 & 0.000 & 0.000 & 0.000 \\
\hline QR & 0.000 & 0.000 & 0.000 & 0.000 \\
\hline \multicolumn{5}{|l|}{ Hogar ampliado } \\
\hline Inferior & -0.081 & -0.022 & -0.013 & -0.043 \\
\hline Superior & 0.107 & 0.038 & 0.036 & 0.063 \\
\hline QR & 0.015 & -0.005 & 0.007 & 0.015 \\
\hline \multicolumn{5}{|l|}{ Hogar unipersonal } \\
\hline Inferior & -0.232 & -0.072 & -0.081 & -0.058 \\
\hline Superior & 0.069 & 0.023 & 0.027 & 0.088 \\
\hline QR & 0.048 & -0.001 & -0.010 & 0.014 \\
\hline
\end{tabular}


Cuadro 6. Continuación.

\begin{tabular}{l|cccc}
\hline \multicolumn{1}{c}{ Variables } & QR20 & QR50 & QR60 & QR90 \\
\hline Número de menores & & & & -0.029 \\
\hline Inferior & -0.058 & -0.027 & -0.036 & 0.011 \\
\hline Superior & 0.025 & -0.001 & -0.005 & -0.025 \\
\hline QR & -0.008 & -0.013 & -0.014 & -0.023 \\
\hline Número de mayores & & & & 0.075 \\
\hline Inferior & -0.022 & -0.018 & -0.008 & -0.002 \\
\hline Superior & 0.099 & 0.048 & 0.042 & 0.005 \\
\hline QR & 0.019 & 0.018 & & -0.136 \\
\hline Localidad urbana & & & -0.048 & -0.031 \\
\hline Inferior & 0.015 & 0.000 & 0.056 & -0.030 \\
\hline Superior & 0.176 & 0.080 & 0.015 & \\
\hline QR & 0.063 & 0.018 & & \\
\hline
\end{tabular}

Fuente: Estimaciones propias con base en microdatos de la ENIGH 2014, INEGI.

*QR corresponde al valor de los coeficientes de la regresión por cuantiles.

\section{CONCLUSIONES}

Esta investigación surge del interés por entender las desigualdades en las tasas de ahorro de los hogares. A partir de los microdatos de la ENIGH 2014, encontramos que los primeros cuatro deciles presentan tasas negativas, en el decil $V$ son cercanas a cero, y los deciles IX y X reportan una mayor capacidad de ahorro. Esto evidencia la necesidad de tomar en cuenta la distribución de la tasa de ahorro en el análisis. En cuanto a los factores explicativos del ahorro de los hogares, con base en la revisión de la literatura, se propuso analizar variables económicas (ingreso permanente, mercados financieros, redes de transferencias y personas ocupadas) y sociodemográficas (sexo, edad, clase del hogar, número de menores, número de mayores y localidad de residencia).

Los resultados de la estimación cuantílica mostraron la importancia de las variables económicas, ya que todas fueron estadísticamente significativas. Por su parte, la edad y la clase del hogar no fueron estadísticamente significativas en las variables sociodemográficas.

Los hallazgos de la investigación proporcionan nueva evidencia para el caso mexicano sobre las diferencias de factores explicativos a lo largo de la distribución de la tasa de ahorro. Destacan las marcadas diferencias entre los cuantiles extremos analizados: QR20 (hogares con tasas de ahorro negativas) y QR90 (hogares con mayores tasas de ahorro). Por ejemplo, la propensión marginal al ahorro en 
el primer grupo de hogares fue de 5.7 por ciento y en el último de 16.1 por ciento. Además, el acceso a los mercados financieros y el sexo del jefe del hogar no fueron significativos en QR20. Otra diferencia fue que el número de personas mayores registró un efecto positivo y significativo sobre la tasa de ahorro de los hogares en QR20, mientras que en QR90 no fue significativo. La localidad urbana tiene un efecto positivo sobre la tasa de ahorro en QR20 y negativo en QR90. En otras palabras, mientras que para el grupo de hogares con tasas de ahorro negativas el acceso a mercados financieros no incentiva el ahorro, para el resto de los hogares son relevantes las oportunidades que brindan estos mercados. El hecho de que la variable sexo no fue estadísticamente significativa muestra que, en efecto, las pautas y patrones de ahorro vinculados al género son diferenciadas entre subgrupos; pero es relevante la contribución de las personas mayores al ahorro en estos hogares. Así, las variables identificadas en cada cuantil muestran los factores que pueden considerarse en el diseño de medidas y acciones cuyo objetivo central es incentivar el ahorro de los hogares.

Finalmente, una posible limitación es la ausencia de información sobre ingreso permanente y la necesidad de incorporar la variable en el modelo a través de estimaciones. Otra limitante es la falta de un método econométrico que permita solucionar los problemas que enfrentan las estimaciones por cuantiles cuando existe endogeneidad; la regresión cuantílica con variables instrumentales ofrece una primera aproximación. Como parte de las futuras líneas de investigación, está pendiente la consideración de nuevas aproximaciones metodológicas y la atención al análisis del desahorro de los hogares.

\section{REFERENCIAS BIBLIOGRÁFICAS}

Attanasio, Orazio P. (1998), "Cohort Analysis of Saving Behavior by U.S. Households", The Journal of Human Resources, 33, pp. 575-609.

Attanasio, Orazio P. y Székely, M. (1999), "Ahorro de los hogares y distribución del ingreso en México", Economía Mexicana, nueva época, 8, pp. 267-338.

Bebczuk, Ricardo; Gasparini, Leonardo; Garbero, Noelia y Amendolaggine, Julian (2015), "Understanding the Determinants of Household Saving: Micro Evidence for Latin America”, en PAPERS, W. (ed.), Argentina, Centro de Estudios Distributivos, Laborales y Sociales (CEDLAS).

Bernal Lara, P. (2007), Ahorro, crédito y acumulación de activos en los hogares pobres de México, Monterrey, Consejo de Desarrollo Social.

Blinder, Alan S. y Deaton, August S. (1985), "The Time Series Consumption Function Revisited”, Brookings Papers on Ecorromic Activity, 2, pp. 465-511. 
Bosworth, Barry; Burtless, Gary y Sabelhaus, John (1991), "The Decline in Saving: Evidence from Household Surveys", Brookings Papers on Economic Activity, Washington, Brookings Institution.

Browning, Martin y Lusardi, Annamaria (1996), "Household Saving: Micro Theories and Micro Facts", Journal of Economic Literature, 34 (4), pp. 1797-1855.

Butelmann, Andera y Gallego, Francisco (2000), "Ahorro de los hogares en Chile: evidencia microeconómica”, Economía Chilena, 3 (1), pp. 5-24. (2001), "Estimaciones de los determinantes del ahorro voluntario de los hogares en Chile (1988 y 1997)", Working Papers Central Bank of Chile, 97, pp. 1-44.

Campbell, John y Mankiw, Gregory (1990), "Permanent Income, Current Income, and Consumption", Journal of Business y Economic Statistics, 8 (3), pp. 265-279.

Ceballos, Owen E. (2015), "Ahorro, crédito y acumulación de activos en presencia de adultos mayores y contingencias económicas", Sobre México. Temas en Economía, 1 (1), pp. 4-24.

Chen, Chien-Liang; Kuan, Chung-Ming y Lin, Chu-Chia (2007), "Saving and housing of Taiwanese households: New evidence from quantile regression analyses", Journal of Housing Economics, 16 (2), pp. 102-126.

Chernozhukov, Victor; Fernández-Val, Iván y Kowalski, Amanda E. (2015), "Quantile regression with censoring and endogeneity", Journal of Econometrics, 186 (1), pp. 201-221.

Chowa, Gina A. N.; Masa D., Rainier y Ansong, David (2012), "Determinants of Saving among Low-Income Individuals in Rural Uganda: Evidence from Assets Africa", Advances in Applied Sociology, 2 (4), pp. 280-291.

Coronado, Julia L. (1998), "The Effects of Social Security Privatization on Household Saving: Evidence from the Chilean Experience", Board of Governors of the Federal Reserve System Finance and Economics Discussion.

De Nardi, Mahacristina; French, Eric y Jones, John B. (2010), "Why Do the Elderly Save? The Role of Medical Expenses", Journal of Political Economy, 118 (1), pp. 39-75.

Deaton, Angus (1992), Understanding Consumption, Oxford, Clarendon. (1997), The Analysis of Households Surveys: A Microeconometric Approach to Development Policy, Baltimore, Johns Hopkins University Press.

Denizer, Ceudet y Wolf C., Holger (1998), "Household Savings in Transition Economies", Policy Research Working Paper.

Duncan, Thomas (1993), "The Distribution of Income and Expenditure within the Household", Center Discussion Paper 669, New Haven, Connecticut, Yale University, Economic Growth Center, 32 pp.

Dynan, Karen E.; Skinner, Jonathan y Zeldes, Stephen P. (2004), "Do the Rich Save More?", Journal of Political Economy, 112 (2), pp. 397-444. 
Espenshade, Thomas J. (1975), "The impact of children on household saving: Age effects versus family size", Population Studies, 29 (1), pp. 123-125.

Flavin, Marjorie A. (1981), "The Adjustment of Consumption to Changing Expectations about Future Income", Journal of Political Economy, 89, pp. 974-1009.

Friedman, Milton (1957), A Theory of the Consumption Function, New Jersey, Princeton University.

Fuentes, Ricardo y Villagómez, Alejandro (2001), "El ahorro en los hogares de bajos ingresos en México: un análisis por cohortes”, El Trimestre Económico, 68-269, pp. 109-133.

Hall, Robert E. (1978), "Stochastic Implications of the Life Cycle-Permanent Income Hypothesis: Theory and Evidence", Journal of Political Economy, 86 (6), pp. 971-987.

Hammer, Jeffrey S. (1986), "Population Growth and Savings in ldcs: A Survey Article”, World Development, 14 (5), pp. 579-591.

Hira Tabira, K. y Loibl, Cäzilia (2008), "Gender Differences in Investment Behavior", en Xiao, Jing J. (ed.), Handbook of Consumer Finance Research, Springer, New York, pp. 253-270.

Hurd, Michael D. (1987), "Savings of the Elderly and Desired Bequest", The American Economic Review, 77 (3), pp. 298-312.

INEGI (2009), ENIGH 2008, Nueva construcción. Ingresos y gastos de los hogares, INEGI.

(2017), Encuesta Nacional de Ingresos y Gastos de los Hogares 2016, Diseño conceptual y definición de categorías y variables.

Khan, Khalid; Ali, Muhammad y Nishat, Mohammed (2010), "Estimation of Consumption function under the PIH: Evidence from Pakistan", Kashmir Economic Review, 19 (1), pp. 91-96.

Liberda, Barbara Z.; Górecki, Brunon y Pęczkowski, Marek (2004), "Saving from Permanent and Transitory Income. The Case of Polish Households", Ekonomia journal, 14, pp. 7-21.

Manitsaris, Athanasios (2006), "Estimating the European Union Consumption Function under the Permanent Income Hypothesis", International Research Journal of Finance and Economics, 2 (3), pp. 1450-2887.

Minh, Nguyen T.; Nhat, Nguyen H.; Anh, Trinh T.; Duc, Phung M. y Son, Le T. (2013), "Demographics and Saving Behavior of Households in Rural Areas of Vietnam: An Empirical Analysis", Journal of Economics and Development, 15 (2), pp. 5-18.

Modigliani, Franco (1986), "Life cycle, individual thrift, and the wealth of nations", The American Economic Review, 76 (3), pp. 297-313. 
Modigliani, Franco y Brumberg, Richard (1954), Utility Analysis and the Consumption Function: an Interpretation of Cross-section Data, New Brunswick, Rutgers University Press.

Montes, Andrés y Villagómez, Alejandro (2002), "El efecto de los hijos sobre el ahorro de los hogares mexicanos", Economía Mexicana, nueva época.

Nava, Isalia; Brown, Flor y Domínguez, Lilia (2013), “¿Hasta qué punto los patrones del ahorro son distintos entre mujeres y hombres?”, en Domínguez, Lilia y Brown, Flor (comps.), México, mujeres y economía, México, UNAM, ONUMUJERES, pp. 119-161.

(2014), "Diferencias de género en los factores asociados al ahorro de los hogares en México", Estudios Demográficos y Urbanos, 29 (2), pp. 301-339.

Poterba, James M. (1994), International Comparisons of Household Saving, Chicago, The University of Chicago Press.

Raut, Laxmi (1989), "Demographic Links to Savings in Life Cycle Models: Identification of Issues for ldcs", The Indian Economic Journal, 40 (1), pp. 116-138.

Rosenzweig, Mark R. (2001), "Savings behavior in low-income countries", Oxford Review of Economic Policy, 17, pp. 40-54.

Seguino, Stephanie y Floro M. Sagrario (2003), "Does Gender have any Effect on Aggregate Saving? An empirical analysis", Internacional Review of Applied Economics.

Solís, Fernando y Villagómez, Alejandro (1999), "Ahorro y pensiones en México: un estudio al nivel de las familias", Economía Mexicana, nueva época, 8 (2), pp. 339-366.

Székely, Miguel (1998), "Monto y distribución del ahorro de los hogares en México", El Trimestre Económico, 65 (2), pp. 263-313.

Tobin, James (1958), "Liquidity Preference as Behavior Towards Risk, Review of Economy Studies, 25 (1), pp. 65-86.

Valles, Yearim y Aguilar, Alberto (2015), "El ahorro en México: evidencia en hogares", Estudios Económicos, CNBV, 3, pp. 41-72.

Vicéns, Otero J. y Sánchez, Reyes B. (2012), "Regresión cuantílica: estimación y contrastes". [Acceso 26/02/2018].

Villagómez, Alejandro y Montes, Andrés (2002), "El efecto de los hijos sobre el ahorro de los hogares mexicanos", Economía Mexicana, nueva época.

\section{AGRADECIMIENTOS}

Esta investigación se realizó en el marco del Proyecto del Consejo Nacional de Ciencia y Tecnología (CONACYT) 255008 "Cambio en la estructura por edades, ahorro y seguridad social en México". 


\section{Anexos}

Cuadro 1. Determinantes del ingreso permanente.

\begin{tabular}{lc}
\hline \multicolumn{1}{c}{ Variables } & MCo \\
\hline Sexo & $0.050^{* * *}$ \\
Edad & $0.033^{* * *}$ \\
Edad $^{2}$ & $0.000^{* * *}$ \\
Primaria & $0.289^{* * *}$ \\
Secundaria & $0.616^{* * *}$ \\
Preparatoria & $0.881^{* * *}$ \\
Licenciatura y más & $1.332^{* * *}$ \\
Perceptores de ingreso & $0.120^{* * *}$ \\
Otros ingresos & $0.000^{* * *}$ \\
Constante & $8.303^{* * *}$ \\
N & 18,191 \\
R-squared & 0.4093 \\
\hline
\end{tabular}

Fuente: Estimaciones propias con base en microdatos de la ENIGH 2014, INEGI. Nota: Estadísticamente significativo al $0.01 * * *, 0.05^{* *}$ y $0.10 *$ 
